\title{
Cardiac Arrest Caused by Torsades de Pointes Tachycardia after Successful Atrial Flutter Radiofrequency Catheter Ablation
}

\author{
Aglaia-Angeliki Mantziari*, Vassilios P. Vassilikos, Yiannis S. Chatzizisis, Georgios Dakos, \\ Georgios Stavropoulos, Stelios Paraskevaidis and Ioannis H. Styliadis
}

$1^{\text {st }}$ Cardiology Department, AHEPA Hospital, Aristotle University of Thessaloniki, Thessaloniki, Greece

\begin{abstract}
A 66-year-old woman underwent successful radiofrequency catheter ablation for long-lasting, drug refractory fast atrial flutter. Two days later she had a cardiac arrest due to torsades de pointes (TdP) tachycardia attributed to relative sinus bradycardia and QT interval prolongation. After successful resuscitation further episodes of TdP occurred, which were treated with temporary pacing. Because of concomitant systolic dysfunction due to ischemic and valvular heart disease she was finally treated with an implantable defibrillator. In conclusion we strongly advise prolonged monitoring for 2 or more days for patients with structural heart disease following successful catheter ablation for long lasting tachyarrhythmias.
\end{abstract}

Keywords: Polymorphic ventricular tachycardia, implantable defibrillator, acquired long QT, ventricular fibrillation.

\section{INTRODUCTION}

Radiofrequency catheter ablation is a safe and effective first line treatment of atrial flutter [1]. Single procedure mean success rate is $92 \%$ and freedom from arrhythmia at 12 months is about $88 \%[2,3]$. Acute complication rate and mortality after radiofrequency catheter ablation of atrial flutter are low (0-0.5\% and 0-0.6\% respectively) [2]. We present a case of cardiac arrest due to torsades de pointes (TdP) tachycardia secondary to relative bradycardia which occurred $48 \mathrm{~h}$ after successful radiofrequency catheter ablation of the cavotricuspid isthmus for long-lasting atrial flutter with fast ventricular response.

\section{CASE REPORT}

A 66-year-old Caucasian woman was referred to our department for electrophysiological testing and possible radiofrequency catheter ablation of atrial flutter. She had long-lasting, unresponsive to pharmacological treatment atrial flutter with 2:1 atrioventricular conduction with a mean heart rate of $120 \mathrm{bpm}$. The patient had a history of ischemic and valvular heart disease with previous coronary artery bypass graft surgery, prosthetic mitral valve replacement and a left ventricular ejection fraction of $25 \%$. There was no reversible myocardial ischemia and the mitral valve was functioning properly. Electrophysiological testing revealed a typical, isthmus dependent atrial flutter. Successful ablation of the cavotricuspid isthmus was performed without any complications and sinus rhythm was restored at $60-70 \mathrm{bpm}$.

*Address correspondence to this author at the $1^{\text {st }}$ Cardiology Department, AHEPA Hospital, Aristotle University of Thessaloniki, 1 Stilponos Kiriakidi Str, 54636, Thessaloniki, Greece; Tel: +306977436678; Fax: +302310994673; E-mail: lmantziari@yahoo.com
After the ablation procedure the patient was transferred to the cardiology ward. On the second day post ablation she had 2 syncopal episodes followed by an episode of cardiac arrest that required a defibrillation shock. ECG recording prior to defibrillation showed coarse ventricular fibrillation. After successful resuscitation the patient was admitted to the coronary care unit and a few hours later an episode of TdP that caused hemodynamic instability was recorded (Fig. 1). Electrocardiographic monitoring revealed relative sinus bradycardia and a long QTc interval $(480 \mathrm{~ms})$ preceding the initiation of TdP. The patient was on ramipril, furosemide, digoxin, acenocoumarol, spironolactone, metformin and sulfonyluria, none of which is related to QT interval prolongation, all serum electrolyte levels were normal and there was no evidence of myocardial ischemia. Older ECGs showed a QTc interval within normal limits. A temporary pacing wire was implanted in order to increase the heart rate and suppress further TdP episodes. Due to the sustained QTc prolongation and the concomitant heart failure the patient was finally treated with an implantable defibrillator and was discharged in a good condition. Five months later she had a defibrillator discharge following a rapid atrial fibrillation event.

\section{DISCUSSION}

Cardiac arrest following atrial flutter ablation is extremely rare [2]. A recent meta-analysis reported no TdP events among 1307 patients who underwent atrial flutter ablation [2]. Polymorphic ventricular tachycardia due to bradycardia-induced QT prolongation after ablation has been previously described after His ablation [4] or ablation of permanent junctional reciprocating tachycardia [5]. In the latter case, the patient had a rapid heart rate for a prolonged period of time before ablation. Similarly, in our case sudden termination of the arrhythmia and deceleration of heart rate 

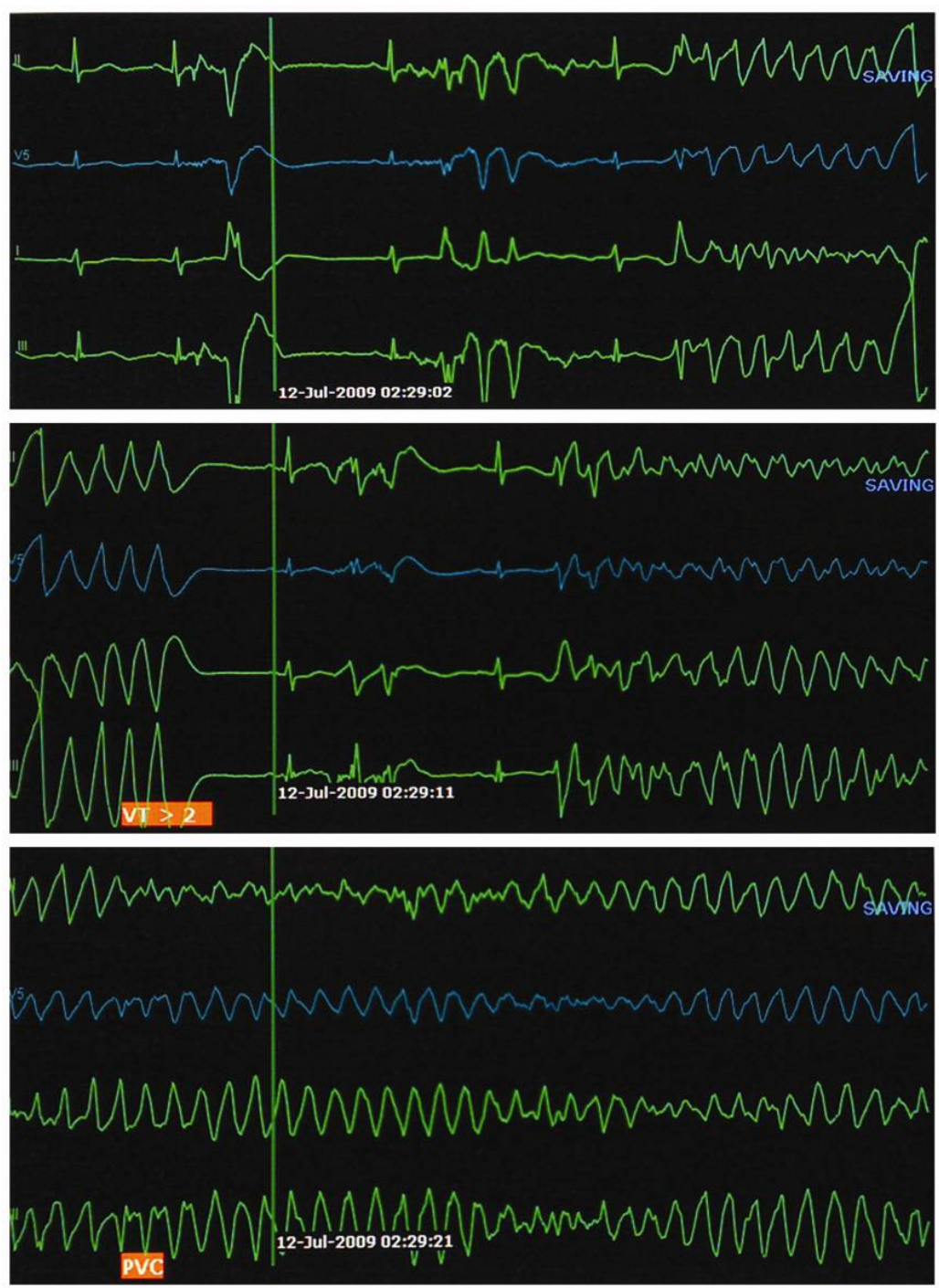

Fig. (1). The electrocardiographic monitor strips show torsades de pointes associated with QT prolongation. Of note, there is a short preinitiating RR interval due to ventricular premature systoles following a long cardiac cycle (long-short phenomenon).

had probably provided the substrate for QT prolongation and TdP induction, even though true bradycardia did not occur. A possible explanation is that the cessation of long-standing high ventricular rate prolongs the ventricular refractory period and presumably the action potential duration at a given cycle [6]. Of note, predisposition to QT prolongation could not be excluded [7]. Ventricular arrhythmias, usually polymorphic, and sudden cardiac death have also been described in patients who are treated with permanent pacing after ablation-induced complete heart block. These arrhythmic events have been attributed to a temporary electrical instability caused by the change in heart rate and the activation sequence, which increase the heterogeneity of repolarization [8]. In such cases, where a permanent pacemaker is required, TdP episodes can be avoided by overdrive pacing at a level above $80 \mathrm{bpm} \mathrm{[8].}$

In our case however, atrioventricular conduction remained intact and permanent pacing after the ablation procedure was not a therapeutic option. Despite the uncomplicated procedure and early course, the patient experienced an aborted sudden death on the second day post ablation. Per- manent pacing at a relatively high rate could suppress further TdP episodes; nevertheless our patient had structural heart disease with systolic heart failure of both ischemic and valvular etiology that places her at a high risk group for sudden cardiac death. Moreover, QTc prolongation did not resolve during the following days. Thus, we decided to implant a cardioverter-defibrillator.

In conclusion, polymorphic ventricular tachycardia might occur after radiofrequency ablation of long-lasting atrial flutter with relative fast heart rate. Prolonged ECG monitoring is advised in such patients in order to avoid similar complications.

\section{REFERENCES}

[1] Sawhney NS, Anousheh R, Chen WC, Feld GK. Diagnosis and management of typical atrial flutter. Cardiol Clin 2009; 27: 55-67.

[2] Spector P, Reynolds MR, Calkins H, et al. Meta-analysis of ablation of atrial flutter and supraventricular tachycardia. Am J Cardiol 2009; 104: 671-7.

[3] Rodgers M, McKenna C, Palmer S, et al. Curative catheter ablation in atrial fibrillation and typical atrial flutter: systematic review and economic evaluation. Health Technol Assess 2008; 12: 1-198. 
[4] Kappos KG, Kranidis AJ, Anthopoulos LP. Torsades de pointes following radiofrequency catheter His ablation. Int J Cardiol 1996; 57: 177-9.

[5] Grimm W, Hoffmann J, Menz V, Maisch B. Transient QT prolongation with torsades de pointes tachycardia after ablation of permanent junctional reciprocating tachycardia. J Cardiovasc Electrophysiol 1999; 10: 1631-5.

[6] Krebs ME, Szwed JM, Shinn T, Miles WM, Zipes DP. Short-term rapid ventricular pacing prolongs ventricular refractoriness in patients. J Cardiovasc Electrophysiol 1998; 9: 1036-42.
[7] Vollmer F, Brembilla-Perrot B, Thiel B. Polymorphous ventricular tachycardia occurring three months following radiofrequency ablation of the bundle of His. Ann Cardiol Angeiol 1998; 47: 109-12.

[8] Nowinski K, Gadler F, Jensen-Urstad M, Bergfeldt L. Transient proarrhythmic state following atrioventricular junction radiofrequency ablation: pathophysiologic mechanisms and recommendations for management. Am J Med 2002; 113: 596-602.

() Mantziari et al.; Licensee Bentham Open.

This is an open access article licensed under the terms of the Creative Commons Attribution Non-Commercial License (http://creativecommons.org/licenses/ by-nc/3.0/) which permits unrestricted, non-commercial use, distribution and reproduction in any medium, provided the work is properly cited. 